\title{
Irradiance Observations of SMM, Spacelab 1, UARS, and ATLAS Experiments
}

\author{
By RICHARD C. WILLSON
}

\begin{abstract}
Solar Irradiance Monitoring Group, Earth and Space Sciences Division, Jet Propulsion
\end{abstract} Laboratory, California Institute of Technology, Pasadena, CA 91109, USA

\begin{abstract}
Detection of intrinsic solar variability on the total flux level was made using results from the first Active Radiometer Irradiance Monitor (ACRIM) experiment, launched on the Solar Maximum Mission (SMM) in early 1980. ACRIM I, specifically designed to start the high precision total solar irradiance database as part of the U.S. Climate Research Program, produced high precision results throughout the 9.75 years of the Solar Maximum Mission. The second ACRIM experiment was flown aboard the Space Shuttle as part of the NASA/ESA Spacelab 1 Mission in late 1983. Its primary function has been to provide a comparison with ACRIM I that could be used to relate its observations with future satellite solar monitors, should they and ACRIM I fail to overlap in time. The second ACRIM satellite solar monitoring experiment (ACRIM II) has provided high precision total solar irradiance observations since its launch as part of the Upper Atmosphere Research Satellite (UARS) mission in late 1991 and continues at present. The shuttle ACRIM instrumentation has been flown on the ATLAS 1 and 2 missions in 1992 and 1993, providing comparisons with the UARS/ACRIM II.
\end{abstract}

\section{The SMM/ACRIM I experiment}

The ACRIM I experiment on the Solar Maximum Mission provided the first unambiguous evidence of intrinsic total solar irradiance (TSI) variability (Willson 1980) (see Figure 1). The first clear evidence was a TSI 'dip' near 1980 day 100 (Willson et. al. 1981; Willson \& Hudson 1981). This was caused by two large sunspot groups in an early stage of their evolution. The TSI increases on either side of this dip were caused by faculae and plage surrounding the sunspots, which are the first and last components of the groups to be seen when they rotated onto and then off our side of the Sun. These two groups were identifiable for six solar rotations. On each successive rotation the ratio of facular area to sunspot area increased within these two groups. The impact of this can be clearly seen during the second dip near day 125 at which time the effect of the sunspot area was significantly offset by the presence of relatively enhanced facular area (Willson 1982, 1984).

The solar cycle TSI variation of about $0.1 \%$ peak-to-peak can be clearly seen in Figure 2 which shows the entirety of the ACRIM I results for the 9.75 years mission. The occurrence of the TSI minimum near the time of solar activity minimum (September 1986), as reckoned by the reversal of polarity of active regions, confirms the solar cycle character of the TSI variation (Willson \& Hudson 1988, 1991). One of the most important aspects of the discovery of a direct proportionality of TSI and solar activity is that its direction is that required for solar forcing of climate change. This makes solar forcing a possible explanation of the 'Spörer, Maunder and Dalton' climate minima that occurred from the 14th through the 19th centuries and the 'Medieval Climate Maximum' of the 12th century (Eddy 1977; Anderson 1991). 


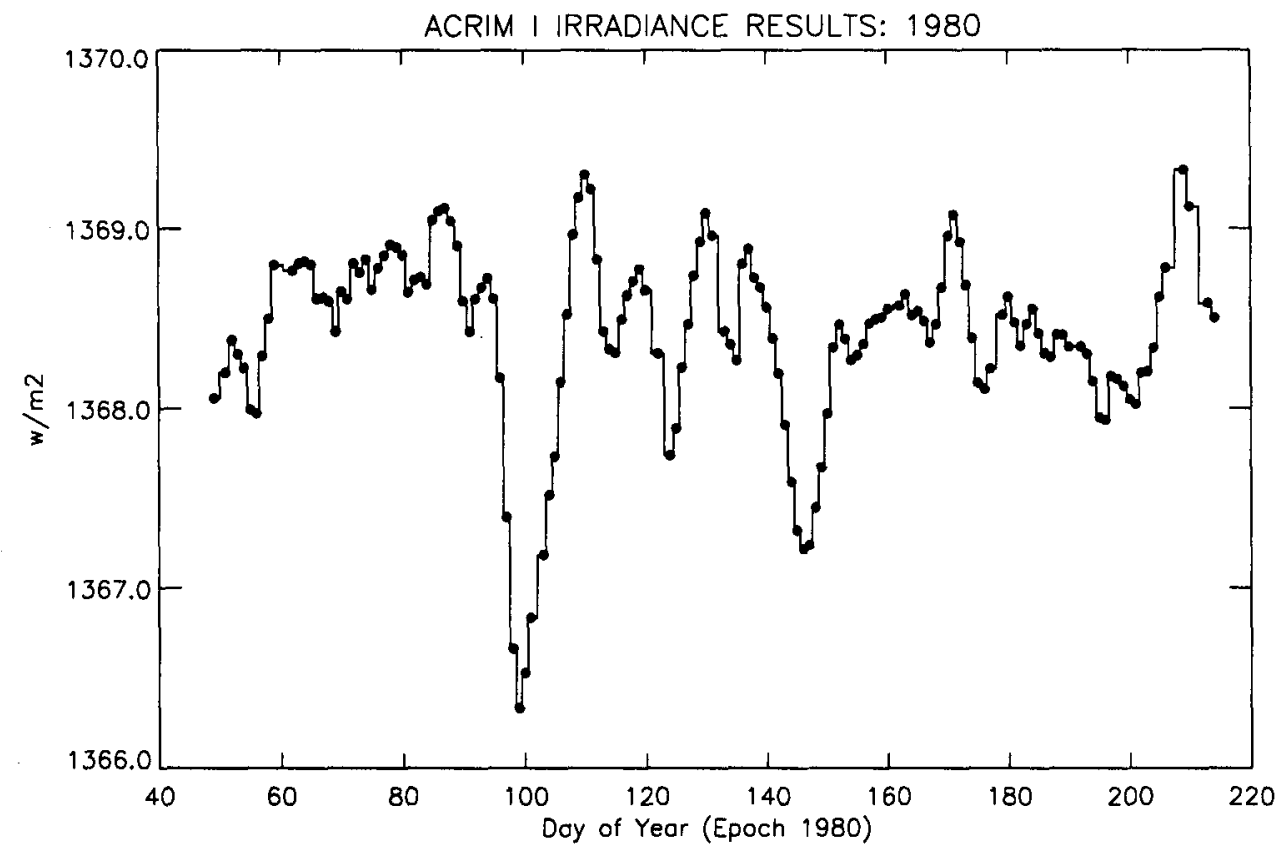

FIGURE 1. Results from the SMM/ACRIM I experiment. Daily mean irradiance values are shown for the first six months of observation.

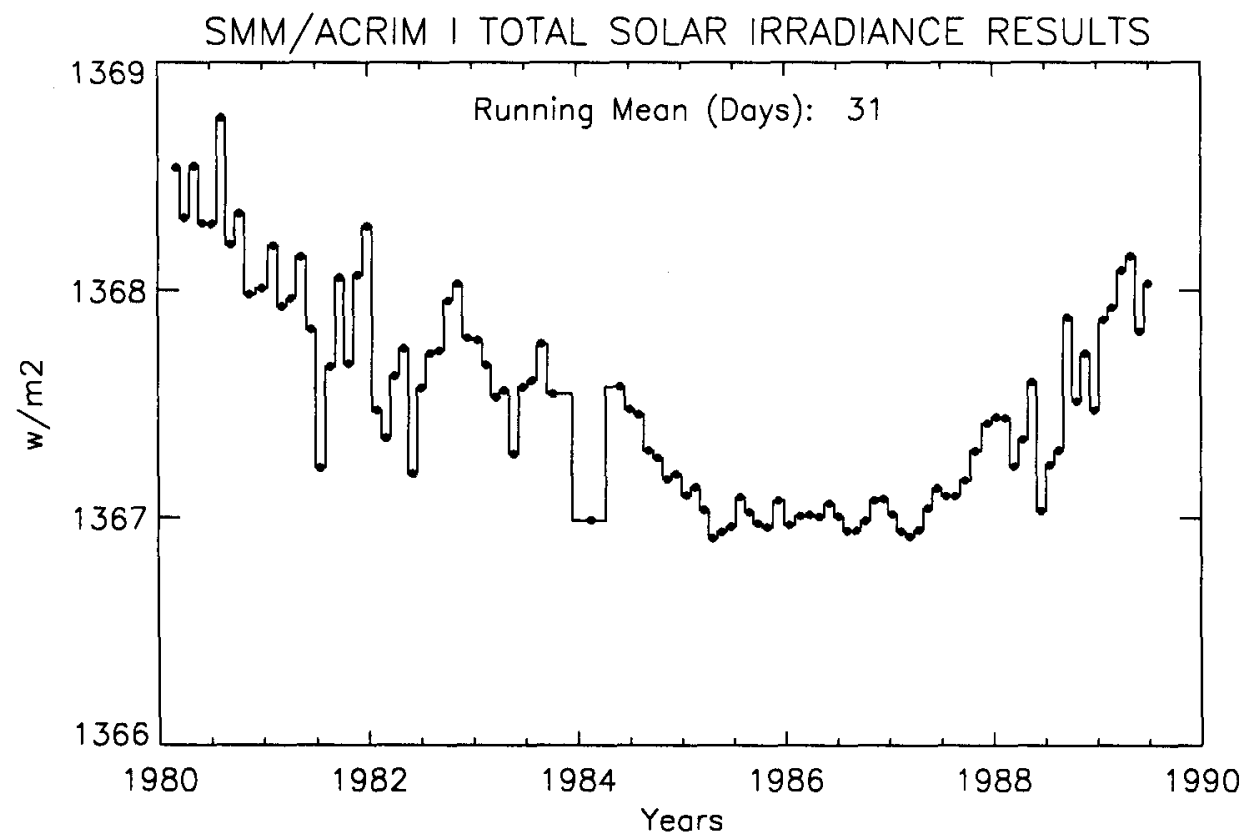

FIgURE 2. Results from the SMM/ACRIM I experiment. 31-day running means smoothing is used to clarify the long term solar cycle trend. 
Comparisons

Nimbus7/ERB - SMM/ACRIM I

Nimbus7/ERB - SMM/ACRIM II

\section{Ratio to Standard Number of \\ ACRIM Error $(\sigma)$ Samples}

$1.003138 \quad 0.000004 \quad 2714$

$\begin{array}{lll}1.004946 & 0.000003 \quad 225\end{array}$

\section{ACRIM I/ACRIM II (ERB Comparisons) $1.001802 \quad 0.000002$}

TABLE 1. Comparisons of SMM/ACRIM I and UARS/ACRIM II with Nimbus-7/ERB experiment. Demonstration of the 'overlap strategy' capability for preserving the precision of the total solar irradiance database.

\section{The UARS/ACRIM II experiment}

The ACRIM II experiment was launched in September 1991, nearly two years after the demise of the Solar Maximum Mission. The results to date are shown in Figure 3. The launch delay of several years, caused by the Challenger accident, prevented the planned direct on-orbit comparisons between ACRIM I and II. The importance of relating their results with precision through direct in-flight comparison cannot be understated since the inherent absolute accuracy of flight TSI sensors is inadequate to sustain a useful long term database. Fortunately, we have been able to relate their results using the next best approach - by comparing both ACRIM I and ACRIM II with the contemporary observations by the Nimbus-7/ERB experiment (Willson 1992). These comparisons are summarized in Table 1 and shown in Figure 4. The benefit of having redundant TSI monitoring experiments in place is clear from this, it has saved the continuity of the TSI database. Without the benefit of these overlapping comparisons, the relationship of future TSI observations to the past could not be established with precision smaller than the solar cycle variation.

From Table 1 it can be seen that the ratio of ACRIM I/ACRIM II can be established with an uncertainty of less than $5 \mathrm{ppm}$, assuming that only random errors affect the results. That this is not quite the case can be clearly seen in Figure 4. Systematic differences are seen in the plot of the ratio of Nimbus-7/ERB to ACRIM I in the form of an approximately sinusoidal variation nearly centered on their 9.45 years set of comparisons. Similarly, a systematic downward trend can be seen in the ratio of Nimbus-7/ERB to ACRIM II.

The causes of systematic differences between ACRIM and ERB are not known with certainty. They may be due to uncorrected thermal effects of the ERB instrumentation during the 1980-1990 period and to uncorrected degradation of ERB's sensitivity during 1991-1992. Other evidences of shorter term ERB sensitivities to thermal effects can be observed in the results as divergences from the means in Figure 4 that significantly exceed the standard errors. The revision of the ERB results by Hoyt \& Kyle (1990) in which earlier database problems due to pointing errors and thermal drifting were significantly reduced, concluded that residual thermal effects remained. A component of the systematic differences is probably contributed by ACRIM I misperformance as well, although by virtue of its more stable spacecraft thermal environment and much higher frequency of electrical self-calibration, the contributions are likely to be much smaller than ERB's.

Similar arguments implicate degradation of ERB's sensitivity as the source of the systematic downward trend relative to the ACRIM II results. By 1992 ERB had been in orbit more than 14 years, and although its total amount of direct solar exposure is only 


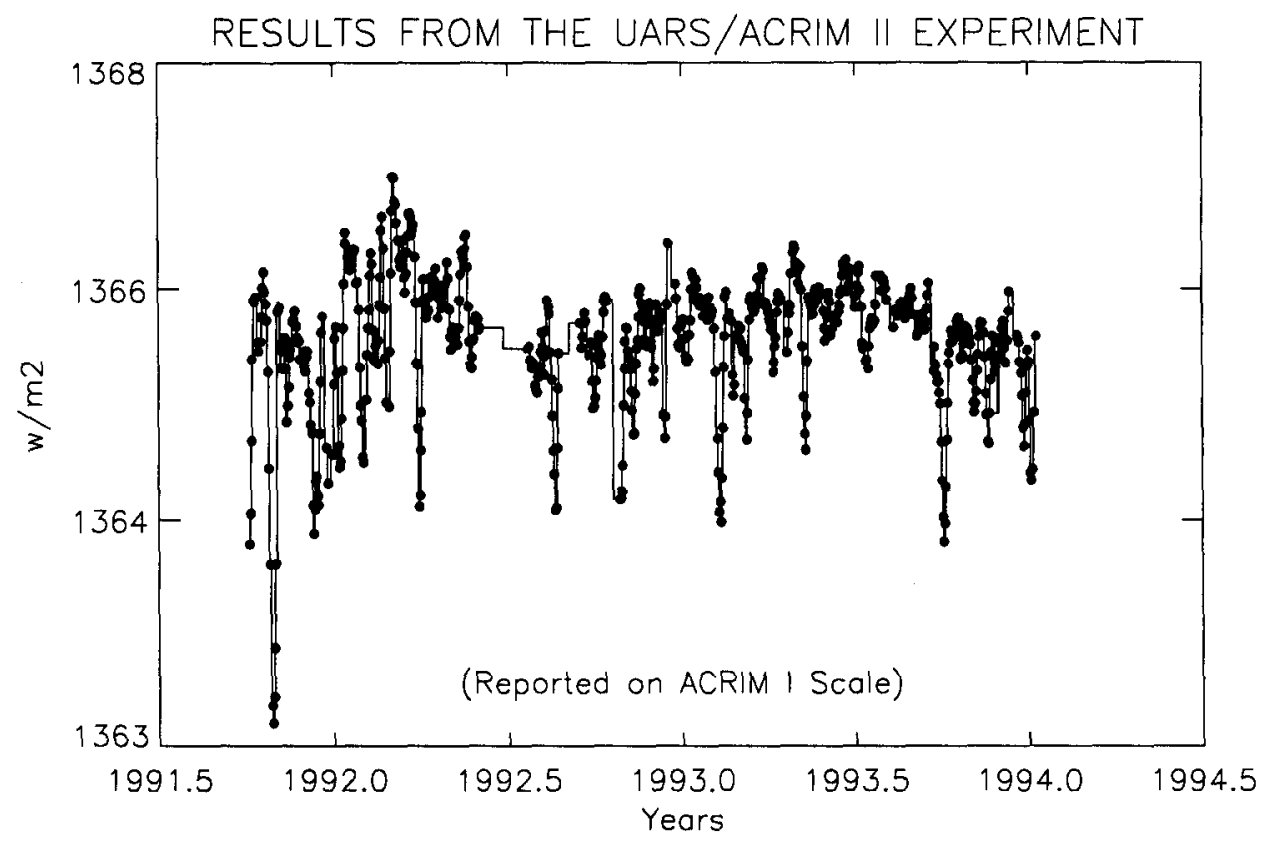

Figure 3. Results from the UARS/ACRIM II experiment. Daily mean irradiances are shown reported on the scale of the SMM/ACRIM I experiment (see Table 1).
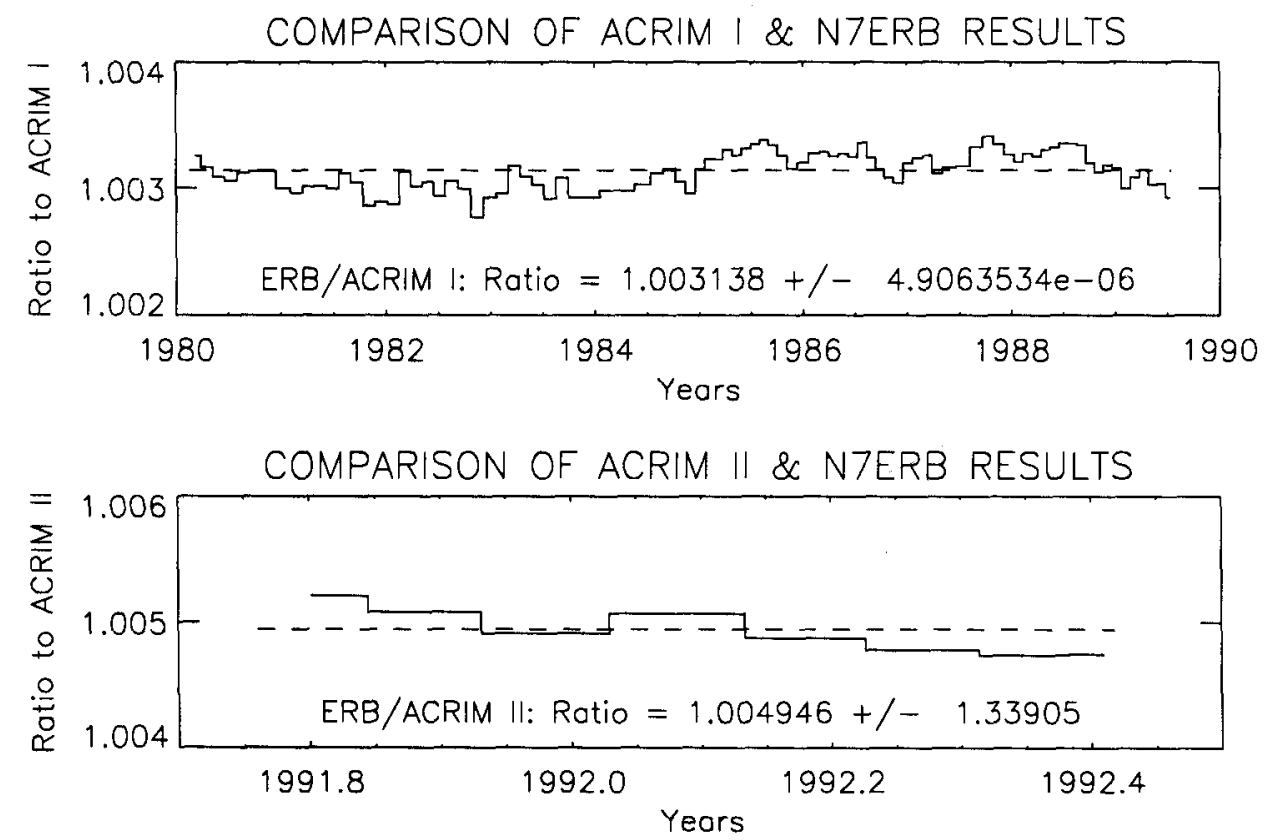

FIgURE 4. Comparisons of SMM/ACRIM I, UARS/ACRIM II and the Nimbus-7/ERB experiments. Results are shown as percentage variation. 
about $10 \%$ of that of the ACRIM's per orbit, degradation should be expected to be a factor in ERB results after that length of time. ERB has no degradation self-calibration capability. The ACRIM experiments have a multi-sensor degradation calibration ability that has demonstrated an inverse relationship of detector sensitivity and exposure to solar direct flux. Therefore this type of relative trend suggests sensitivity degradation of the ERB, particularly during this period of exceptionally high solar activity when enhanced levels of high energy solar flux and particles are present, as the cause of the relative trend.

A more realistic estimate of the uncertainties of the ERB/ACRIM comparisons is being pursued through minimization of the systematic effects by modeling. Preliminary results indicate the residual uncertainty of the ACRIM I/ACRIM II ratio will be greater than the $2 \mathrm{ppm}$ of Table 1 and smaller than 50 .

\section{Spacelab 1/ACRIM, ATLAS/ACRIM and contemporary total solar irradiance experiments}

Several TSI experiments have been conducted since launch of the UARS/ACRIM II. The first was the ATLAS1/ACRIM in March 1992, the second the ATLAS2/ACRIM experiment in April. Two ESA TSI experiments, the PMOD (Brusa \& Fröhlich 1972) and CROM (Crommelynck 1981) were included on the EURECA platform which was launched by the Shuttle in 1992 and retrieved in 1993 (see also Crommelynck et. al. 1994; Fröhlich 1994; Romero et. al. 1994).

The shuttle-based ACRIM experiment flown on the ATLAS 1 and 2 missions was also flown on the Spacelab 1 mission in December 1983. The principal purpose of the shuttle ACRIM experiment was to make underflight comparisons with satellite TSI monitoring experiments so that if the latter failed to overlap the precision of the database could be sustained through mutual inter comparisons with the shuttle ACRIM. The results of comparisons with satellite experiments are shown in Table 2. The Spacelab 1/ACRIM data were acquired during the SMM/ACRIM I mission. The ATLAS 1 and 2/ACRIM results were acquired during the UARS/ACRIM II experiment.

In the interest of understanding the reliability of the shuttle ACRIM results, the ACRIM II observations are scaled to ACRIM I as discussed previously, to facilitate comparison of Spacelab 1 and ATLAS measurements. Sensor ACR502 was included in the ACRIM instrument on all three shuttle flights, the ACR504 on two. The weighted means are dominated by the ATLAS results because of their smaller uncertainties. This is caused by far smaller number of observations acquired during the Spacelab 1 mission when near total failure of the shuttle command-and-data system (RAU) occurred. The spread of the observations from the flights ( $\pm 242 \mathrm{ppm}$ for ACR502 and $\pm 152 \mathrm{ppm}$ for ACR504) exceeds their statistical uncertainties by a large margin, indicating the clear intervention of sources of systematic error. It has been observed that the clean cavities of the ACRIM sensors provided for integration in each mission are contaminated when returned for recalibration after each flight. Contamination is believed to be the principal cause of the variation of results from flight-to-flight. The largest spread is between the ACR502 result of ATLAS 1 and the other two, indicating that its second flight result could be anomalously high. This may be resolved by the ATLAS-3/ACRIM experiment in late 1994.

It was hoped that the shuttle ACRIM series would be able to provide mission-to-mission precision better than $100 \mathrm{ppm}$ instead of the approximately $200 \mathrm{ppm}$ observed from the results to date. While larger than expected, this bound of uncertainty is an order of magnitude superior to the absolute uncertainty of the current generation of sensors, that 


\begin{tabular}{|c|c|c|c|c|}
\hline Mission & $\begin{array}{l}\text { Sensor } \\
\text { ACR502 } \\
\text { Average } \\
\text { (\% var) }\end{array}$ & $\begin{array}{c}\text { Uncertainty } \\
\text { Std. Error }(\sigma) \\
\pm \\
(\% \text { var })\end{array}$ & $\begin{array}{l}\text { Sensor } \\
\text { ACR504 } \\
\text { Average } \\
\text { (\% var) }\end{array}$ & $\begin{array}{c}\text { Uncertainty } \\
\text { Std. Error }(\sigma) \\
\pm \\
(\% \text { var })\end{array}$ \\
\hline Spacelab $1(12 / 83)$ & 0.3679 & 0.0098 & 0.4479 & 0.0088 \\
\hline ATLAS $1(3 / 92)$ & 0.4164 & 0.0028 & Not Flown & - \\
\hline ATLAS $2(4 / 93)$ & 0.3680 & 0.0006 & 0.4174 & 0.0007 \\
\hline $\begin{array}{l}\text { Weighted Mean } \\
\text { (All flights) }\end{array}$ & 0.3701 & 0.0006 & 0.4176 & 0.0007 \\
\hline Variation $( \pm)$ & 0.0242 & & 0.0152 & \\
\hline $\begin{array}{l}\text { Weighted Mean } \\
\text { (ATLAS } 1 \text { \& } 2 \text { ) }\end{array}$ & 0.3701 & 0.0006 & - & - \\
\hline Variation $( \pm)$ & 0.0242 & & & \\
\hline $\begin{array}{l}\text { Weighted Mean } \\
\text { (SL } 1 \text { \& ATLAS 2) }\end{array}$ & 0.3680 & 0.0010 & 0.4176 & 0.0011 \\
\hline Variation $( \pm)$ & 0.0022 & & 0.0152 & \\
\hline
\end{tabular}

TABLE 2. Results from the Space Shuttle ACRIM experiment on the Spacelab 1, ATLAS 1 and ATLAS 2 missions. Results are shown for ACR sensors 502 and 504 as their percentage variation relative to the radiation scale defined by the SMM/ACRIM I instrument.

operate at 'ambient temperature' and comparable to the absolute uncertainty of new cryogenic TSI sensors currently under development. From this standpoint, the shuttle ACRIM experiment is a valuable insurer of the long term TSI database. Should overlap of satellite TSI monitoring experiments fail to occur in the future, the shuttle ACRIM comparisons could be used to sustain the TSI database precision at a useful level, even if less precisely than had been hoped.

The ESA EURECA platform was launched from the Shuttle in mid-1992 and retrieved about nine months later. Two TSI monitors resided on the platform: the PMOD developed by Fröhlich (see Romero et al. 1994) and CROM developed by Crommelynck (see Crommelynck et al. 1994). A comparison of preliminary daily mean results obtained simultaneously by the PMOD, CROM and the UARS/ACRIM II experiments is shown in Figure 5 (Fröhlich 1993; Crommelynck 1993). The EURECA data shown begins shortly after its deployment. The results from all three experiments depict essentially the same principal TSI variations, including the 'sunspot deficit' effect of large sunspot groups in the early and late part of the period. The ACRIM II and PMOD results are most closely matched in both SI units and the shape of their time series. The CROM utilizes a different operational mode and differs significantly in design from the PMOD and ACRIM which probably explains the differences of shape of its time series. Both sets of EURECA results demonstrate higher initial decreases in flux, relative to ACRIM II. This is probably due to high rates of degradation of their cavity absorbing surfaces in the early observations, a common feature of the initial performance of TSI sensors in space. The dashed lines of Figure 5 occur during periods when the UARS spacecraft was inoperational due to failure of the solar array's pointing mechanism and no simultaneous results were available for comparison.

These same data are presented in Figure 6 as the percentage difference from the UARS/ACRIM II results. A general downward trend in both the relative sets of results probably indicates uncorrected degradation in the PMOD and CROM sensors accumu- 


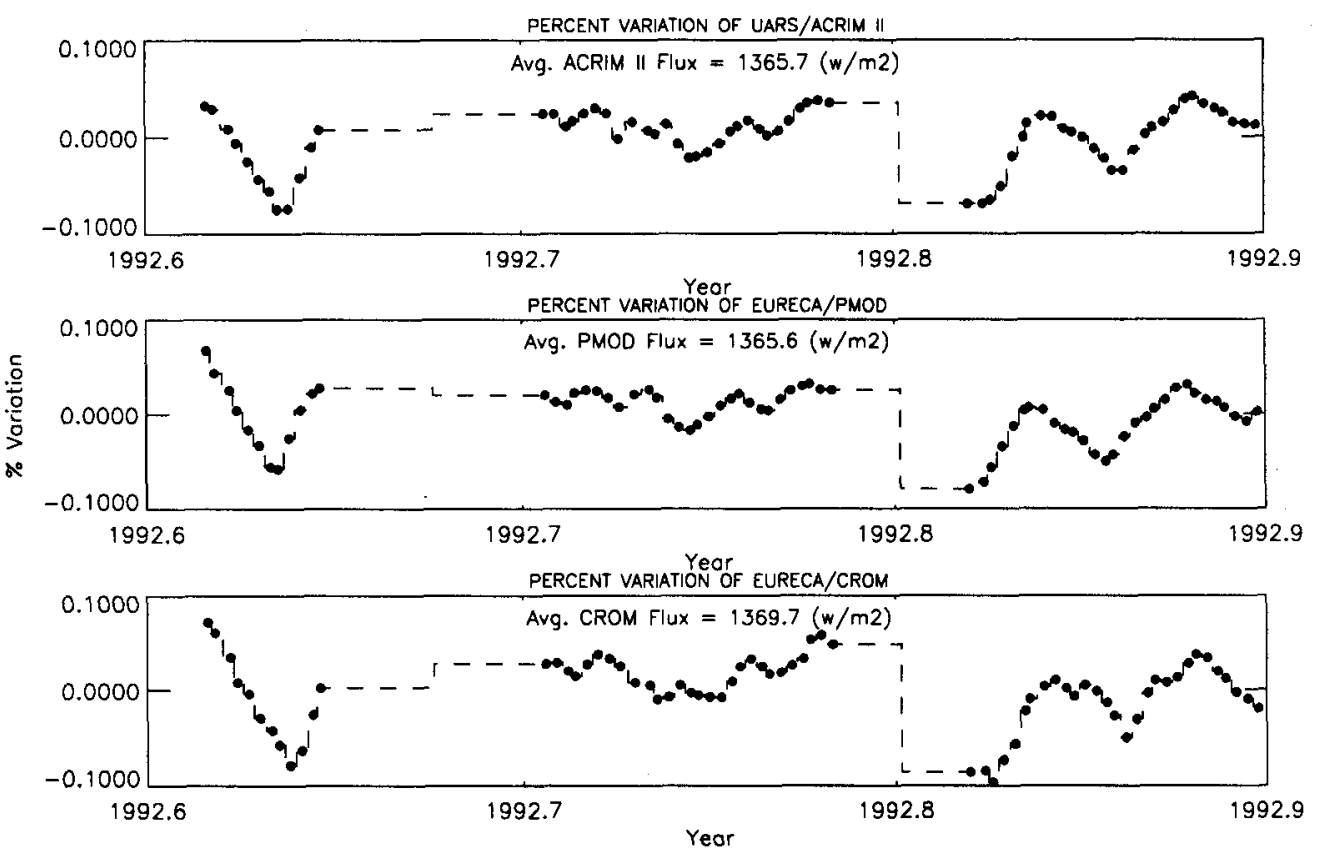

FIgURE 5. Comparisons of UARS/ACRIM II and the EURECA/PMOD and CROM results

lating with exposure to solar high energy fluxes and particles. A high initial rate of relative decline for the CROM may indicate an accelerated initial degradation similar to that found in early results from the ACRIM experiments.

\section{Summary}

The TSI variability detected thus far is directly proportional to solar activity, as would be required by a solar forcing function for climate change that might have been responsible for the 'Maunder or Spörer climate minima' of the 14th through the 17th centuries, and the 'Medieval Climate Maximum' of the 12th century. This implies the need to extend the high precision TSI database indefinitely with maximum available precision to detect subtle, long term TSI variation. A careful measurement strategy that relates successive solar monitoring experiments at a precision level of the instrumentation will be required to sustain the database since the uncertainty of TSI instrumentation on an absolute basis, even if operated at cryogenic temperatures, is inadequate for this purpose.

There are presently no U.S. experiments planned for the mid-to-late 1990's that have a high degree of probability for sustaining the TSI database until deployment of the next ACRIM experiment as part the Mission to Planet Earth (on the Earth Observation System 'Chemistry' satellite in late 2002). If the current experimental scenario persists, the first high precision TSI database will terminate in the late 1990's and the next will begin in 2002 , with effectively no relationship between them of a sufficiently high precision to be useful for the long term study of either solar or climate variability.

Acknowledgments. The research described in this paper was completed by the Jet Propulsion Laboratory, California Institute of Technology, under a contract with the 

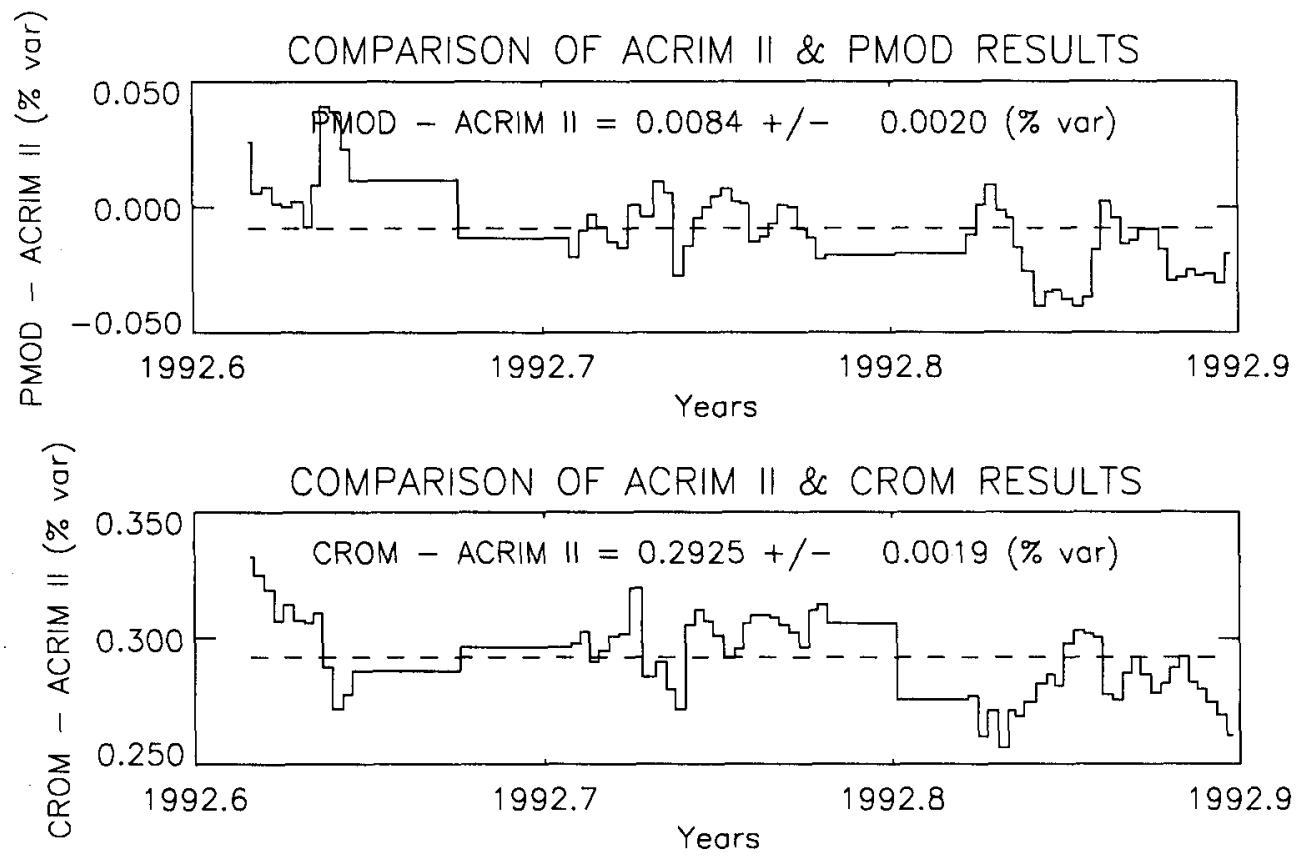

FIGURE 6. Comparison of UARS/ACRIM II with PMOD and CROM results as percentage differences in time series.

National Aeronautics and Space Administration. The author thanks C. Fröhlich and D. Crommelynck for preliminary EURECA/SOVA results.

\section{REFERENCES}

ANDERSON, R.Y. 1991 Solar variability captured in climatic and high-resolution paleoclimatic and high-resolution paleoclimatic records: A geological perspective. In The Sun in Time (ed. C.P. Sonett, M.S. Giampapa \& M.W. Mathews). pp. 543-561. Univ. Arizona Press, Tucson, AZ, USA.

BrusA, R.W. \& Fröhlich, C. 1972 Entwicklung eines neuen Absolutradiometers. Technical Note 1. World Radiation Center, Davos, Switzerland.

Crommelynck, D. 1981 Fundamentals of absolute pyrheliometry and objective characterization. Technical Note World Radiation Center, Davos, Switzerland.

Crommelynck, D. 1993 Private communications.

Crommelynck, D., Domingo, V., Fichot, A. \& Lee, R. 1994 Irradiance observations from the EURECA and ATLAS experiments. In The Sun as a Variable Star: Solar and Stellar Irradiance Variations (ed. J.M. Pap, C. Fröhlich, H.S. Hudson \& S.K. Solanki). Cambridge University Press, in press.

EDDY, J.A. 1977 Historical evidence for the existence of the solar cycle. In Solar Output and Its Variation (ed. O.R. White). pp. 51-71, Univ. of Colorado Press, Boulder, CO, USA.

FRöHLICH, C. 1993 Private communications.

FröHLICH, C. 1994 Reviews of space observations of total solar irradiance. In The Sun as a Variable Star: Solar and Stellar Irradiance Variations, (ed. J.M. Pap, C. Fröhlich, H.S. Hudson \& S.K. Solanki). Cambridge Univ. Press, in press.

HoYT, D. \& KYLE, L. 1990 An alternative derivation of the Nimbus-7 total solar irradiance variations. In Climate Impact of Solar Variability (ed. K.H. Schatten \& A. Arking). NASA 

CP-3086, 293-300.

Romero, J., Wehrli, C. \& Fröhlich, C. 1994 Solar total irradiance variability from SOVA2 on board EURECA. Solar Phys., in press.

Willson, R.C. 1980 Solar irradiance observations from the SMM/ACRIM experiment. American Geophysical Union, Toronto, Canada, May 1980.

Willson, R.C. 1982 Solar irradiance variations and solar activity. J. Geophys. Res. 86, 43194326.

Willson, R.C. 1984 Measurements of solar total irradiance and its variability. Space Science Reviews 38, 203-242.

WILlson, R.C. 1992 Solar total irradiance monitoring, past, present and future. EOS Transactions, American Geophysical Union 73(14), pp. 242.

Willson, R.C. \& Hudson, H.S. 1981 Variations of solar irradiance. Astroph. J. Lett. 24, 185-189.

Willson, R.C. \& Hudson, H.S. 1988 Solar luminosity variations in solar cycle 21. Nature 332, 810-812.

Willson, R.C. \& Hudson, H.S. 1991 The Sun's luminosity over a complete solar cycle. Nature 351, 42-44.

Willson, R.C., Gulkis, S., Janssen, M., Hudson, H. S. \& Chapman, G. A. 1981 Observations of solar irradiance variability. Science 211, 700-702. 